\title{
Uma proposta experimental de eletricidade com o uso da placa de prototipagem Arduino para o ensino de física
}

\author{
An experimental proposal for electricity using the Arduino prototyping board for teaching physics \\ Una propuesta experimental de electricidad utilizando la placa de prototipos Arduino para la \\ enseñanza de física
}

Recebido: 24/01/2021 | Revisado:24/01/2021 | Aceito: 29/01/2021 | Publicado: 07/02/2021

\author{
Antônio de Lisboa Coutinho Júnior \\ ORCID: https://orcid.org/0000-0001-7270-7759 \\ Instituto Federal de Educação, Ciência e Tecnologia do Ceará, Brasil \\ E-mail: lisboajr@gmail.com \\ Jeirla Alves Monteiro \\ ORCID: https://orcid.org/0000-0002-9329-5930 \\ Instituto Federal de Educação, Ciência e Tecnologia do Ceará, Brasil \\ E-mail: jeirla@gmail.com \\ Darkson Fernandes da Costa \\ ORCID: https://orcid.org/0000-0002-8304-0723 \\ Instituto Federal de Educação, Ciência e Tecnologia do Ceará, Brasil \\ E-mail: darksonifce@gmail.com \\ Gilvandenys Leite Sales \\ ORCID: https://orcid.org/0000-0002-6060-2535 \\ Instituto Federal de Educação, Ciência e Tecnologia do Ceará, Brasil \\ E-mail: denyssales@gmail.com
}

\begin{abstract}
Resumo
$\mathrm{O}$ uso do Arduino em laboratórios de física vem crescendo a cada ano, pois é um aparelho pequeno, funcional, com um custo razoavelmente baixo e de fácil acesso. Por tanto, o presente artigo, tem como objetivo propor uma atividade experimental, para o ensino de Eletricidade, que aborda os conceitos da associação de resistores: em série, em paralelo e misto, de forma prática. Utilizando a placa de prototipagem programável Arduino, como aparato emulador de um Ohmímetro digital. Através do instrumento desenvolvido é possível à aquisição de dados, bem como o desenvolvimento de uma série de experimentações, servindo de alternativa aos instrumentos de medição existentes em laboratórios de escolas e instituições de ensino, estabelecendo uma relação estreita entre a Física e a programação. A sugestão de aula pode ser expandida e implementada para outros instrumentos de medição, como o multímetro, amperímetro, voltímetro entre outros, assim como explorada em atividade colaborativa entre alunos e projetos didáticos.
\end{abstract}

Palavras-chave: Atividade experimental; Ensino de física; Arduino; Eletricidade.

\begin{abstract}
The use of Arduino in physics laboratories is growing every year, as it is a small, functional device, with a reasonably low cost and easy access. In this article, we propose a suggestion for the teaching of Electricity, which understand the concepts of the association of resistors: series, parallel and mixed, in a practical way. Using the programmable Arduino prototyping board, as an emulator of a digital Ohmmeter. Through the developed instrument it is possible to acquire data as well as the development of a series of experiments, serving as an alternative to existing measurement instruments in schools and educational institutions, establishing a close relationship between physics and programming. The suggestion can be expanded and implemented for other measuring instruments, as well as explored in collaborative activity between students and didactic projects.
\end{abstract}

Keywords: Experimental activity; Physics teaching; Arduino; Electricity.

\section{Resumen}

El uso de Arduino en los laboratorios de física ha ido creciendo año tras año, ya que es un dispositivo pequeño, funcional, con un costo razonablemente bajo y de fácil acceso. Por ello, en este artículo se propone una s actividad experimental para la enseñanza de la Electricidad, que aborda los conceptos de asociación de resistencias: en serie, en paralelo y mixto, de manera práctica. Usando la placa de creación de prototipos programable Arduino, como un emulador de un ohmímetro digital. A través del instrumento desarrollado es posible adquirir datos, así como el desarrollo de una serie de experimentos, sirviendo como alternativa a los instrumentos de medición existentes en los 
laboratorios escolares e instituciones de enseñanza, estableciendo una estrecha relación entre la Física y la programación. La sugerencia de lección se puede ampliar e implementar para otros instrumentos de medida, como el multímetro, amperímetro, voltímetro entre otros, así como explorar en actividad colaborativa entre estudiantes y proyectos didácticos.

Palabras clave: Actividad experimental; Enseñanza de la física; Arduino; Electricidad.

\section{Introdução}

O progresso e a socialização das tecnologias digitais de informação e comunicação (TDIC) provocaram mudanças comportamentais na sociedade (Silva, 2017). A adição de recursos tecnológicos na esfera da aprendizagem, por intermédio de atividades experimentais de laboratório, pode auxiliar na harmonização dos alunos com o aprender e estimular habilidades para as demandas do mercado de trabalho com as carreiras cada vez mais tecnológicas. Nesse sentido, o modelo de ensino híbrido, que inclui as TDIC nas práticas pedagógicas é uma alternativa viável para contribuir no atendimento das demandas educacionais e sociais da atualidade (Silva, 2017).

A adição de TDIC no ensino de ciências, como as simulações virtuais e experimentos reais, podem propiciar melhores condições para a análise e observação de fenômenos científicos. A utilização das ferramentas tecnológicas já faz parte da rotina da maior parte dos indivíduos, no entanto, percebe-se a dificuldade em se aproximar do cotidiano escolar, formando profissionais com currículos desatualizados, desmotivados e insatisfeitos.

É essencial salientar que, incluir TDIC em práticas pedagógicas não é uma solução para todos os problemas no ensino, é preciso levar em consideração a metodologia do professor e o contexto do aluno (Silva et al., 2018). Nesse sentido, achar que o uso das TDIC representa uma estratégia transformadora do ensino em sala de aula é impreciso. As tecnologias digitais podem integrar e facilitar o aprender por conta própria, a troca rápida de informações, favorecendo feedbacks imediatos e a comunicação, possibilitando que o ensinar e o aprender aconteça de forma natural, favorecendo uma autonomia e colaboratividade dos indivíduos que podendo aprender no seu tempo e no seu espaço.

Outro desafio para o ensino é a respeito da transposição didática (Chevallard, 1991) dos fenômenos, teorias e a matemática formal na construção de leis da física como um todo desde física clássica até a física contemporânea. Os desafios não são exigidos apenas pela complexidade intrínseca dos tópicos da física, mas também por uma insegurança inerente a qualquer tentativa de mudança no domínio escolar impedindo qualquer tipo de inovação (Brockington \& Pietrocola, 2016).

Atualmente, o ensino de física vem ganhando destaque com às pesquisas realizadas que implementam simulações virtuais ou experimentos reais de laboratório como objeto de aprendizagem. A utilização de tecnologias como o arduíno, phet interactive simulations, scratch e entre outras ferramentas podem proporcionar descobertas autênticas, o aprender fazendo (Learn by doing), e gerando dados imediatos através dos fenômenos do ensino de física, apresentando os resultados em tempo real proposto por Sokoloff et al. (2007) como Física em Tempo Real (RealTime Physics). Através da Física em Tempo Real obtém-se excelentes possibilidades de abordagens didáticas que tendem a levar o estudante a sentir-se estimulado e envolvido ativamente na sua própria aprendizagem (Rocha et al., 2014).

Uma das competências gerais proposta pela Base Nacional Comum Curricular (BNCC), direciona-se para exercitar a curiosidade intelectual e recorrer à abordagem própria das ciências, incluindo a investigação, a reflexão, a análise crítica e a criatividade, para investigar causas, podendo ser implementado o uso de TDIC em sala (Brasil, 2019). Nesse sentido, a presente pesquisa tem o objetivo de descrever uma proposta de experimento real com suporte na ferramenta arduino para o ensino de eletricidade. 


\section{Ensino de Física}

Há um tempo que o ensino de física vem sendo executado por apresentação de leis, conceitos, lista de conteúdo, exercícios repetitivos de memorização ou automação, fórmulas em situações artificiais onde a linguagem matemática é desvinculada do significado físico, o que faz com que a física se torne algo distante do mundo vivido tanto dos professores como dos alunos (Veiga, 2015).

Conforme Robilotta e Babichak (1997) ensinar física é difícil, pois a física possui características muito peculiares, que abrangem diferentes áreas: a própria física, que engloba um amplo conhecimento e envolve ideias cada vez mais abstratas sobre uma parte do mundo natural

A Física é vista como algo sem significado e totalmente desassociado da realidade. No Brasil ela ainda é apresentada em um ensino que a demonstra como uma ciência compartimentada, segmentada, pronta, acabada, imutável (Nardi, 2004), e isso contribui para que os alunos pensem que tudo já foi descoberto e que não há nada mais a resolver. A dificuldade de mudança desse quadro tradicional não decorre somente do despreparo dos professores, nem das deficiências e limitações das escolas, mas de uma deformação estrutural.

Diante deste problema na visão sobre o ensino de física, surgem em 1999 os Parâmetros Curriculares Nacionais (PCN e PCN+) que apresentam o conhecimento de Física no ensino médio com uma meta de construir uma visão da Física que esteja voltada para a formação de um cidadão contemporâneo, atuante e solidário, com instrumentos para compreender, intervir e participar da realidade. Algumas das habilidades e competências que, segundo os PCN, o aluno deve desenvolver com o ensino de física são:

1) Compreender enunciados que envolvam códigos e símbolos físicos. Compreender manuais de instalação e utilização de aparelhos.

2) Utilizar e compreender tabelas, gráficos e relações matemáticas gráficas para a expressão do saber físico. Ser capaz de discriminar e traduzir as linguagens matemática e discursiva entre si.

3) Desenvolver a capacidade de investigação física. Classificar, organizar, sistematizar. Identificar regularidades. Observar, estimar ordens de grandeza, compreender o conceito de medir, fazer hipóteses, testar.

4) Conhecer e utilizar conceitos físicos. Relacionar grandezas, quantificar, identificar parâmetros relevantes. Compreender e utilizar leis e teorias físicas.

5) Compreender a Física presente no mundo vivencial e nos equipamentos e procedimentos tecnológicos. Descobrir o "como funciona" de aparelhos.

6) Reconhecer o papel da Física no sistema produtivo, compreendendo a evolução dos meios tecnológicos e sua relação dinâmica com a evolução do conhecimento científico.

Uma das habilidades esperadas que os alunos desenvolvam diz respeito aos meios tecnológicos e sua importância para a evolução do conhecimento científico, sendo assim nas próximas seções serão abordadas o uso do Arduino em um experimento para demonstrar o funcionamento de circuitos elétricos, agindo assim como um meio de integração das tecnologias com o dia a dia escolar dos alunos.

\section{Resistores}

Um dos primeiros componentes da eletrônica, assim como os capacitores, válvulas e indutores; o resistor (Figura 1) é peça fundamental na confecção de uma diversidade de dispositivos eletrônicos e circuitos integrados digitais. 
Figura 1 - Resistor comum.

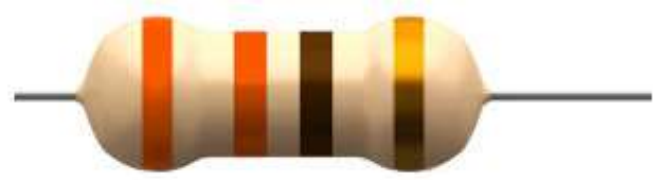

Fonte: https://brasilescola.uol.com.br/o-que-e/fisica/o-que-sao-resistores.htm.

Constituído por hastes metálicas e um invólucro contendo certa substância de baixa condutividade, tais como uma liga metálica de Ni-Cr ou puramente uma fita de carbono. Possui uma grande variedade de modelos, sendo os mais comuns aqueles de filamento metálico e faixas de cores indicadoras de valores conforme indicada na Figura 2.

Figura 2 - Tabela de valores para resistores.

\begin{tabular}{|c|c|c|c|c|c|}
\hline Cor & 1á Faixa & 2a Faixa & 3a Faixa & Multiplicador & Tolerância \\
\hline Preto & $\mathbf{0}$ & $\mathbf{0}$ & $\mathbf{0}$ & $\mathbf{x} \mathbf{\Omega}$ & \\
\hline Marrom & $\mathbf{1}$ & $\mathbf{1}$ & $\mathbf{1}$ & $\mathbf{x} 10 \Omega$ & $+/-1 \%$ \\
\hline Vermelho & 2 & 2 & 2 & $\times 100 \Omega$ & $+/-2 \%$ \\
\hline Laranja & 3 & 3 & 3 & $\times 1 \mathrm{~K} \Omega$ & \\
\hline Amarelo & 4 & 4 & 4 & $\times 10 \mathrm{~K} \Omega$ & \\
\hline Verde & 5 & 5 & 5 & $\times 100 \mathrm{~K} \Omega$ & $+/-.5 \%$ \\
\hline Azul & 6 & 6 & 6 & $\times 1 \mathrm{M} \Omega$ & $+/-25 \%$ \\
\hline Violeta & 7 & 7 & 7 & $\times 10 \mathrm{M} \Omega$ & $+/-.1 \%$ \\
\hline Cinza & 8 & 8 & 8 & & $+/-.05 \%$ \\
\hline Branco & 9 & 9 & 9 & & $+1-5 \%$ \\
\hline Dourado & & & & $\times .1 \Omega$ & $+/-10 \%$ \\
\hline Prateado & & & & $\times .01 \Omega$ & \\
\hline
\end{tabular}

Fonte: https://athoselectronics.com/codigo-de-cores-de-resistores/.

Os resistores podem ainda possuir valores de resistência: fixos (resistores comuns), variados (potenciômetros e trimpots) e inversamente proporcional à tensão recebida em suas terminações (varistores ou VDR - Voltage Dependent Resistor). Podendo ser associado em uma ampla combinação de estruturas, contudo todas seguem três formatos fundamentais, sejam: em série, em paralelo ou de forma mista.

$\mathrm{Na}$ associação em série a Resistência Equivalente $\left(\mathrm{R}_{\mathrm{eq}}\right)$ corresponde a soma de todos os resistores contido na associação:

$$
\mathrm{R}_{\mathrm{eq}}=\mathrm{R}_{1}+\mathrm{R}_{2}+\mathrm{R}_{3}+\mathrm{R}_{4}+\ldots+\mathrm{R}_{\mathrm{n}}
$$

Nesse tipo de associação o valor da resistência equivalente é sempre maior do que as resistências. Essa associação de resistores pode ser representada como mostra a Figura 3.

Figura 3 - Associação de resistores em série.

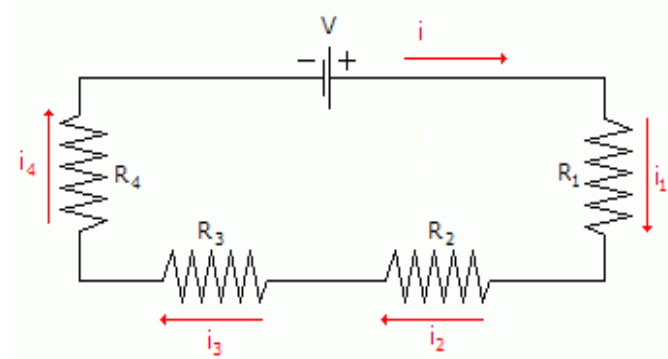

Fonte: https://www.infoescola.com/fisica/associacao-de-resistores/. 
Nesse tipo de associação a Corrente elétrica (I) será sempre igual em todos os resistores, sendo assim pode-se dizer que:

$$
\mathrm{I}=\mathrm{i}_{1}=\mathrm{i}_{2}=\mathrm{i}_{3}=\mathrm{i}_{4} \ldots \mathrm{i}_{\mathrm{n}}
$$

A Tensão (V) obtida poderá ser calculada a partir do somatório de todas as tensões dos respectivos resistores:

$$
\mathrm{V}=\mathrm{V}_{1}+\mathrm{V}_{2}+\mathrm{V}_{3}+\mathrm{V}_{4} \ldots \mathrm{V}_{\mathrm{n}}
$$

A diferença de potencial é calculada pela primeira Lei de OHM, que diz que a diferença de potencial entre dois pontos de um resistor é proporcional à corrente elétrica que é estabelecida nele:

$$
\mathrm{V}=\mathrm{R} \times \mathrm{I}
$$

A Associação em Paralelo acontece quando os resistores são ligados de modo que a corrente elétrica se divide ao passar por eles. Essa associação pode ser representada como mostra a Figura 4.

Figura 4 - Associação em paralelo.

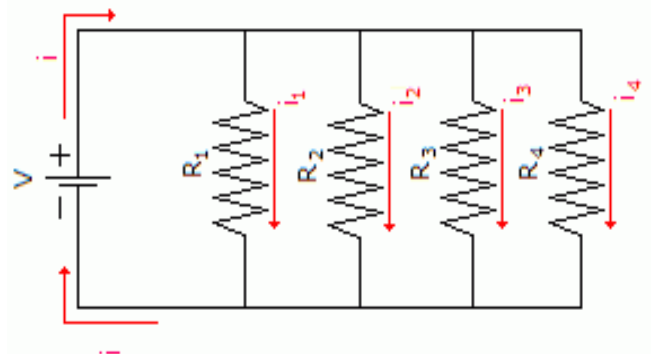

Fonte: https://www.infoescola.com/fisica/associacao-de-resistores/.

Nessa associação, a resistência elétrica equivalente será sempre menor do que a menor das resistências, e pode ser calculada da seguinte forma:

$$
\frac{1}{R e q}=\frac{1}{R 1}+\frac{1}{R 2}+\frac{1}{R 3}+\cdots+\frac{1}{R n}
$$

Na associação em paralelo à Tensão (V) obtida em cada resistor é igual:

$$
\mathrm{V}=\mathrm{V}_{1}=\mathrm{V}_{2}=\mathrm{V}_{3}=\mathrm{V}_{4}
$$

A Corrente (I) será o somatório de intensidade de cada resistor:

$$
\mathrm{I}=\mathrm{i}_{1}+\mathrm{i}_{2}+\mathrm{i}_{3}+\mathrm{i}_{4}
$$


Conforme Maya (1979) o modelo de associação mista é a combinação de circuitos em série e paralelo. Para obter a Resistência Equivalente $\left(\mathrm{R}_{\mathrm{eq}}\right)$ é necessário primeiro calcular a resultante das estruturas em paralelo e posteriormente equacionar os valores postos em série.

\section{Placa de Prototipagem Arduino}

Segundo Banzi (2012) define a placa de prototipagem Arduino (Figura 5) como uma plataforma de computação física de fonte aberta, com base em uma placa simples de entrada/saída, assim como seu ambiente de desenvolvimento. De origem Italiana, é considerado o primeiro hardware livre. A placa é constituída por um conjunto de portas digitais, analógicas e plugins para transmissão de corrente elétrica e/ou troca de informações. Ainda conforme o autor:

[...] o Arduino é formado por dois componentes principais: a placa Arduino, elemento de hardware com o qual você trabalha ao construir seus objetos; o IDE do Arduino, o software que você executa em seu computador. O IDE pode ser utilizado para criar um sketch (esboço, um pequeno programa de computador), do qual você fará um upload para a placa Arduino[...] (Banzi, 2012, p. 33).

Figura 5 - Placa Arduino UNO R3.

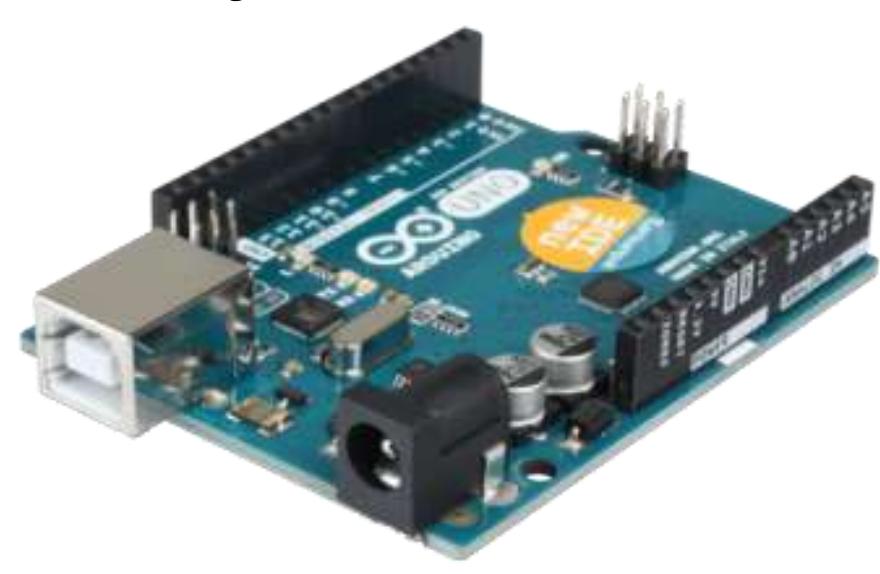

Fonte: www.arduino.cc

As portas da placa, possibilitam tanto coletar como transmitir informações para módulos, sensores e atuadores. Sendo os sensores componentes eletrônicos que captam dados do meio, tais como: temperatura, calor, luminosidade, campo magnético, pressão, movimento etc. Os atuadores, são dispositivos nos quais podemos atuar, isto é, exercer alguma ação física, por exemplo: mover um motor elétrico, ligar uma lâmpada ou um LED, emite um som e/ou acionar um laser. Por fim os módulos são placas circuitadas que podem conter também sensores, bem como outros componentes eletrônicos para transmissão de dados, caracterizam-se como exemplares: módulos Bluetooth, módulos WiFi, módulo coletor de partículas do ar, módulo de comunicação em rede de computadores etc.

A plataforma Arduino possui um ambiente integrado de desenvolvimento, denominado IDE (Integrated Development Environment ou Ambiente de Desenvolvimento Integrado) onde são escritos códigos, funções de controle e instruções para manipular, coletar e transmitir dados para os sensores e atuadores, de acordo com a Figura 6. 
Figura 6 - IDE (Ambiente de Desenvolvimento Integrado).

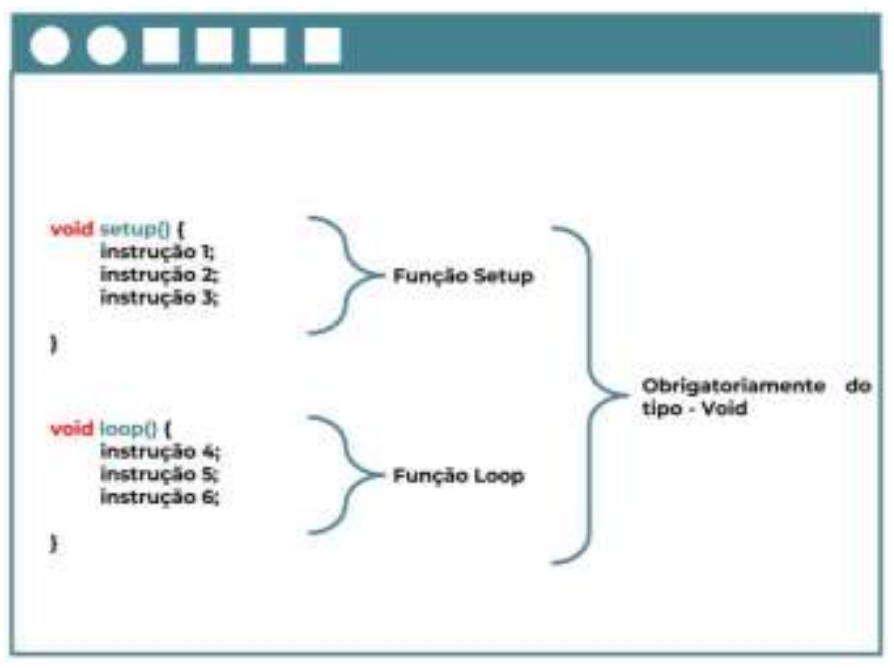

Fonte: Autores.

Dentro da IDE há um painel com o qual] podemos enviar mensagens de controle, bem como receber informações, o chamando Monitor Serial, identificado através de um ícone em formato de lupa. Quando acionado, é aberta uma janela conforme a Figura 7, onde estabelecemos uma conexão direta com o Arduino.

Figura 7 - Monitor Serial.

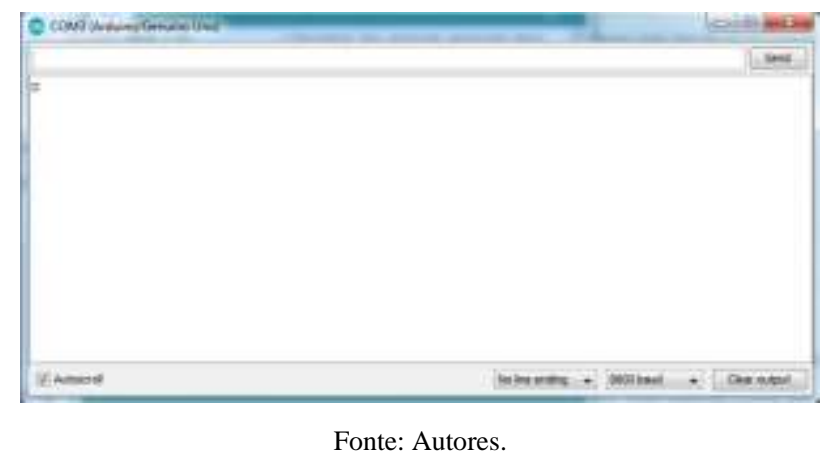

Dentro do painel iremos visualizar o valor da resistência ou do conjunto associado, servindo dessa forma com display. A caixa ao lado do botão Send, será utilizada durante o experimento para inserir o valor do resistor de referência, componente este que será explicado na próxima secção.

\section{Metodologia}

A presente pesquisa tem como percurso metodológico uma abordagem qualitativa, apoiada pela constituição do estado de conhecimento (Morosini, 2015). A escolha deste apoio deu-se, pois, a identificação, síntese e reflexão sobre um conteúdo já produzido, pode contribuir para construção da atividade experimental com a placa de prototipagem Arduino. O desenvolvimento dessa atividade experimental foi feito pensando em aulas de física em laboratórios, de forma com que essas aulas possam ficar mais atrativas para os alunos, com o uso das tecnologias. A construção da atividade experimental ocorreu em duas etapas. A primeira que foi a elaboração do código arduino para o divisor de tensão. A segunda foi sugerir uma sequência didática para desenvolver a atividade com os alunos. 


\subsection{Divisor de tensão}

Para desenvolver um Ohmímetro elementar no Arduino utiliza-se uma montagem eletrônica com resistores associados em série (também denominado: Divisor de Tensão), na modelagem do circuito podemos observar na Figura 8 o esquema adotado.

Figura 8 - Divisor de Tensão.

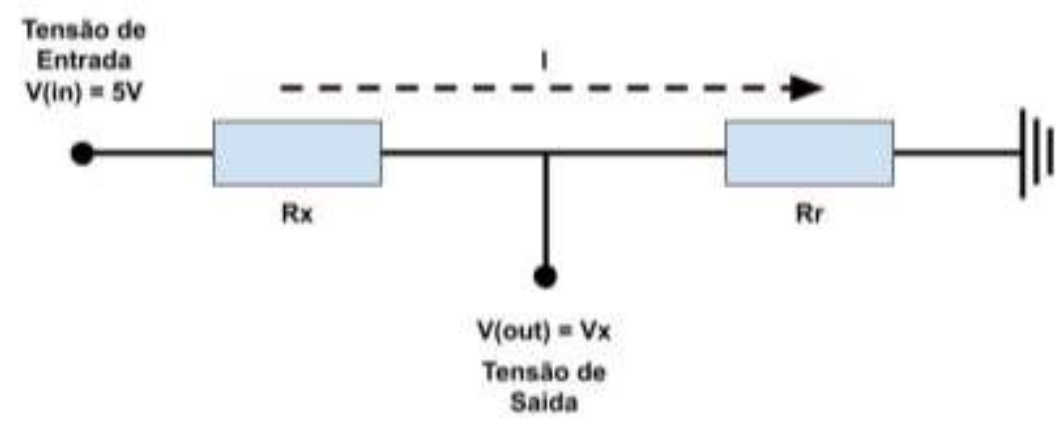

Fonte: Autores.

Na qual I é a corrente contida no circuito, Vx é definida como a tensão que o Arduino irá medir, 5 Volts a tensão de entrada que a placa Arduino fornece (sendo sempre constante), Rr é um valor de resistência já conhecido antecipadamente (é uma resistência que será tomada como referência) e Rx o valor da resistência ainda não encontrado. Aplicando a primeira Lei de Ohm a resistência Rr, obtemos: I = Vx / Rr. Bem como a Lei de Kirchoff sobre resistor Rx: V - Vx = Rx x I.

$$
\begin{gathered}
I=\frac{V_{x}}{R_{r}}(a) \\
V-V_{x}=R_{x} \times I(b)
\end{gathered}
$$

Substituímos o valor de I de (a) na fórmula de (b), e obteremos:

$$
\begin{gathered}
V-V_{x}=R_{x} \times I \Rightarrow V-V_{x}=R_{x} \times \frac{V_{x}}{R_{r}} \Rightarrow \\
V=R_{x} \times \frac{V_{x}}{R_{r}}+V_{x} \Rightarrow V=\left(\frac{R_{x}}{R_{r}}+1\right) \times V_{x} \Rightarrow \frac{V}{V_{x}}=\frac{R_{x}}{R_{r}}+1 \Rightarrow \\
\frac{R_{x}}{R_{r}}=\frac{V}{V_{x}}-1 \Longrightarrow R_{x}=\left(\frac{V}{V_{x}}-1\right) \times R_{r} \Rightarrow \\
R_{x}=\frac{V \times R_{r}}{V_{x}}-R_{r}(c)
\end{gathered}
$$

Por conseguinte, tendo os valores de $\mathrm{V}$ e Rr, podemos realizar a medição de $\mathrm{V}$, resultando na obtenção do valor corresponde ao resistor Rx.

\subsection{Código Arduino}

As seguintes instruções constituem o código necessário para o Arduino atuar de forma semelhante a um Ohmímetro (Quadro 1). A placa será mantida conectada a um PC, Notebook ou Minicomputador (SBC - Single Board Computers ${ }^{1}$ ),

1 SBC (Single Board Computers) ou Computadores de Placa Única são computadores totalmente constituído e confeccionado dentro de uma única placa de circuitos. Com dimensões que não ultrapassam um cartão de crédito. 
através de um cabo USB. Este mesmo cabo irá fornecer a energia necessária para alimentar tanto o Arduino, quanto o experimento de eletricidade.

Quadro 1 - Código de comando Arduino.

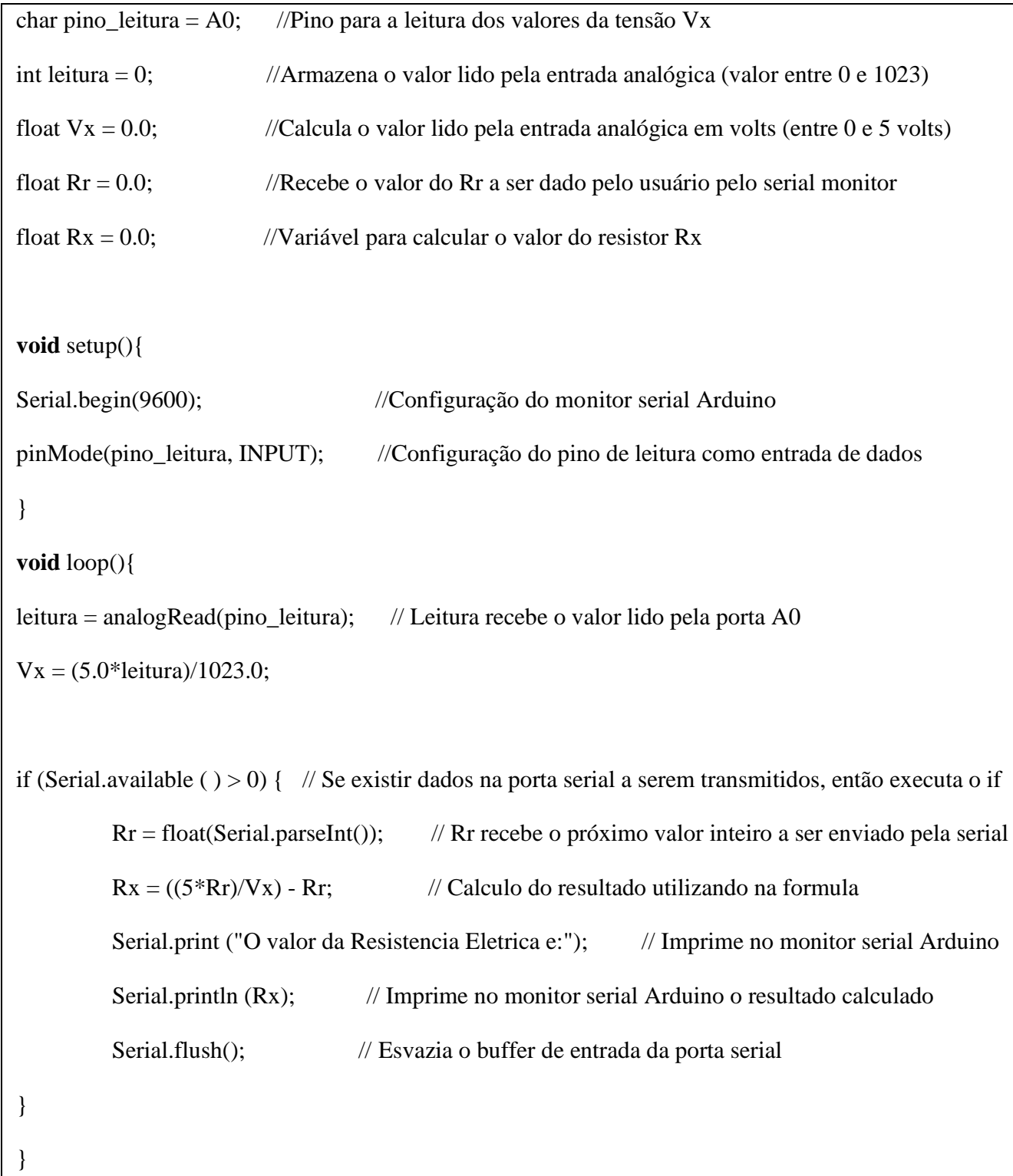

Fonte: Autores.

\subsection{Sequência Didática}

Para Iniciar a sequência didática o aluno deverá executar determinadas tarefas, seguindo um roteiro estabelecido por três fases: I - primeira fase de calibração, II - segunda fase de medição e a III - terceira e última fase constituída por desafios de conhecimento. Cada participante deve receber um kit contendo uma placa Arduino, uma matriz de contatos (protoboard), um conjunto de resistores, um guia de orientação para o manuseio da matriz de contatos, bem como uma lista com modelos de circuitos.

A nota de aula deverá conter uma série de montagens com resistores em série, em paralelo e misto, bem como desafios de maior complexidade. Na primeira fase, de posse da nota de aula base e do código Arduino, o estudante deverá:

(a) copiar o código descrito para o seu ambiente de desenvolvimento integrado (IDE) 
(b) verificar o código e executar/encaminhar para dentro da placa

(c) montar o circuito base conforme a Figura 9.

Figura 9 - Circuito Base.

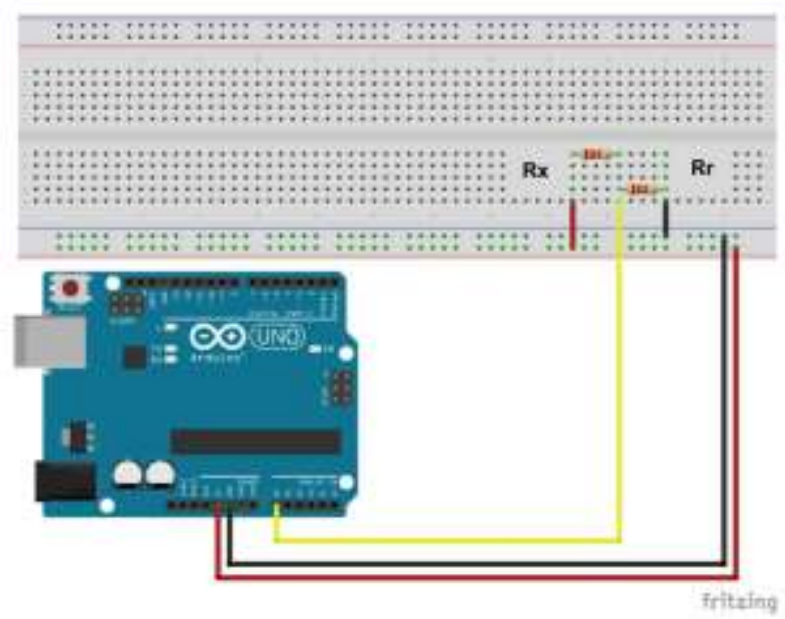

Fonte: Autores.

(d) ler o código de cores do resistor de referência $(\mathrm{Rr})$

(e) abrir o Monitor Serial e digitar o valor indicado pelo resistor de referência

(f) verificar o valor de retorno

Os passos indicados servirão para calibrar o sistema e familiarizar os alunos a estrutura do circuito base.

Na segunda fase são apresentados exemplos de circuitos em série e paralelo. Determina-se um ciclo de aprendizado que será utilizado pelos alunos para cada modelo de circuito estudado. Conforme modelo sugerido por (Rocha \& Guadagnini, 2014, p. 129):

[...] são introduzidos ao tema de estudo e ao projeto do experimento; - são questionados sobre as suas previsões com respeito aos resultados que devem ser obtidos no experimento; - são incentivados a interagirem com seus pares (pequenos grupos) no sentido de discutir as previsões sobre os resultados experimentais; - efetuam o experimento e observam os resultados, em geral representados na forma de tabelas e gráficos gerados em tempo real utilizando sensores e sistemas de aquisição automática de dados; - confrontam os resultados obtidos com as previsões feitas anteriormente, e tentam explicar os fenômenos observados. São desafiados a prever o comportamento de sistemas físicos diferentes dos abordados nos experimentos, mas que são baseados nos mesmos conceitos básicos estudados.

Na Figura 10 é demonstrado dois exemplos de montagens em série, que os alunos deverão fazer. 
Figura 10 - Circuito em Série.

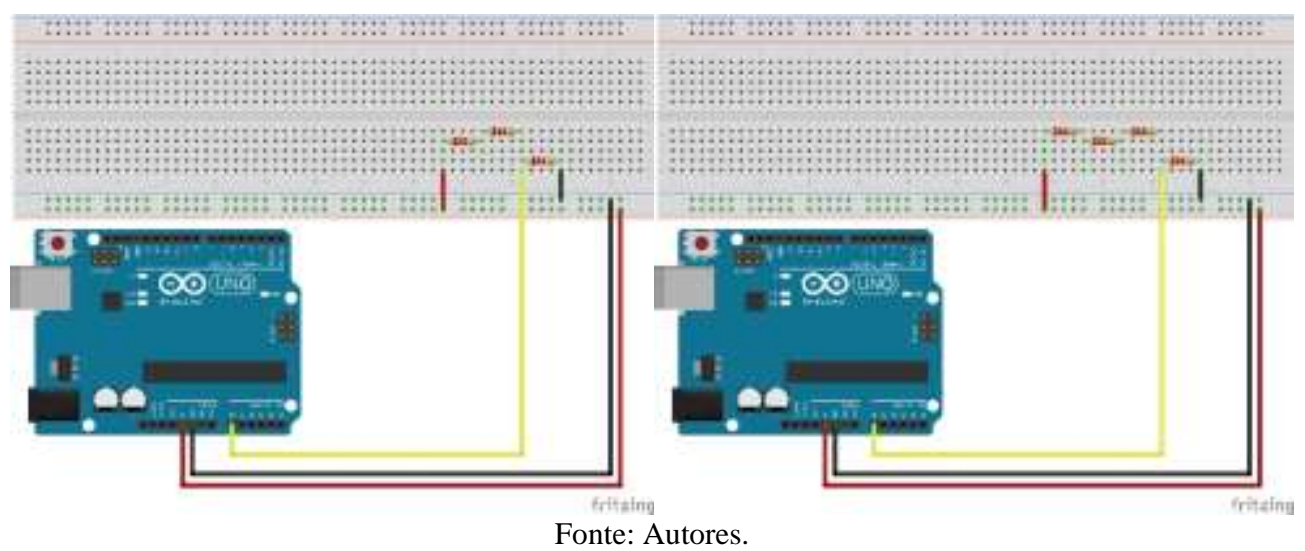

Na Figura 11 contêm dois exemplos de montagem em paralelo que deverão ser feitas pelos alunos.

Figura 11 - Circuito em paralelo.

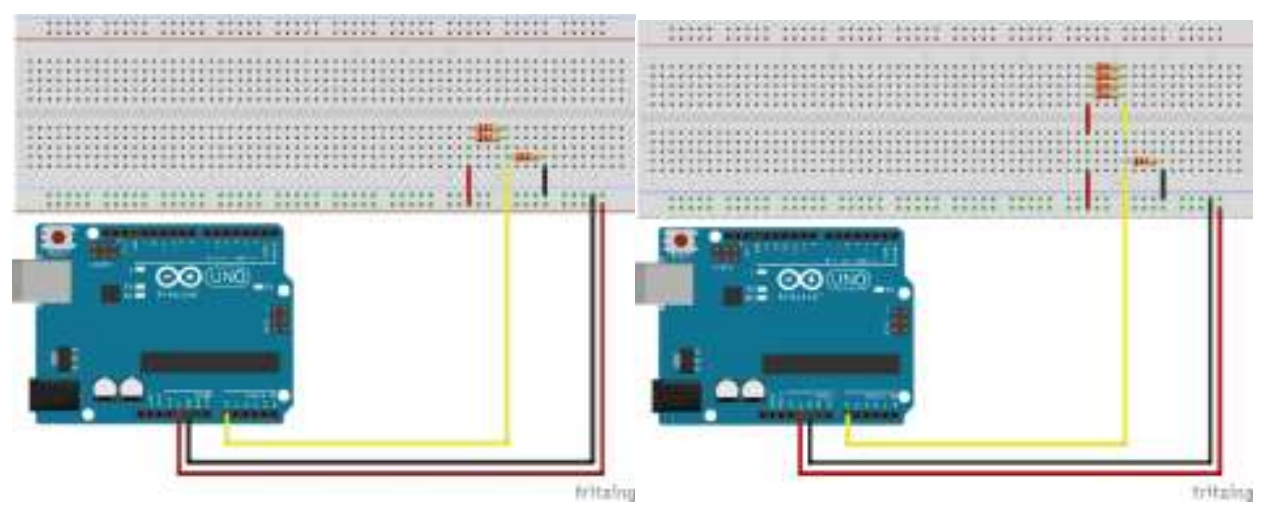

Fonte: Autores.

Já a Figura 12 exibe dois tipos de circuitos mistos, para os alunos reproduzirem na aula.

Figura 12 - Circuito Misto.

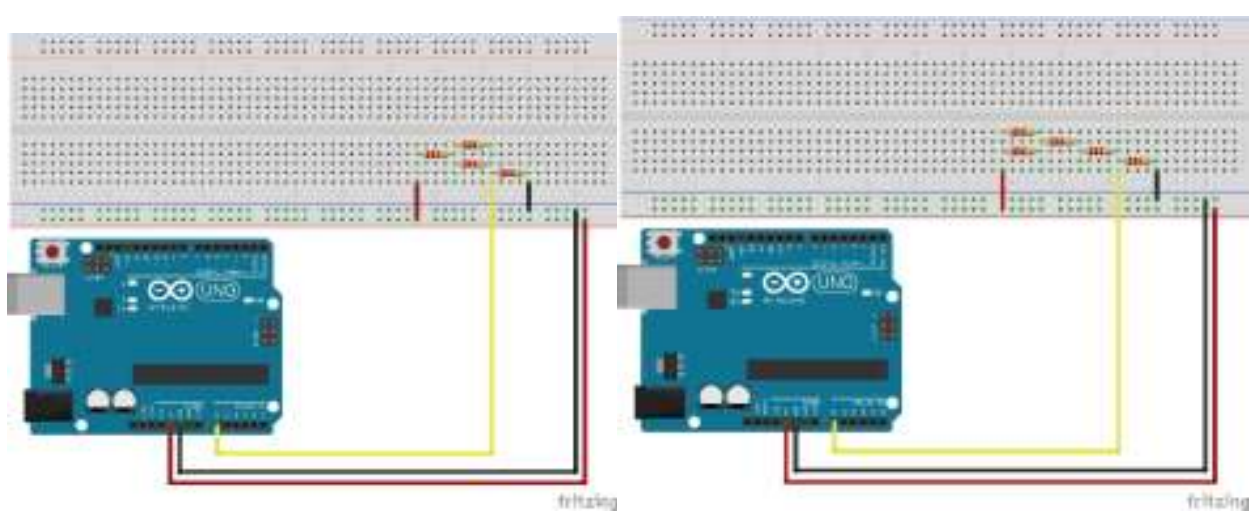

Fonte: Autores.

$\mathrm{Na}$ terceira fase são apresentados desafios contendo circuitos de maior complexidade, com uma variedade de combinações. As montagens podem ser posteriormente armazenadas em um repertório de soluções, que podem ser e aproveitadas em revisões, avaliações, seminários ou apresentações científicas. Na figura 13 observa-se dois exemplos de montagens complexas. 
Figura 13 - Montagens complexas.

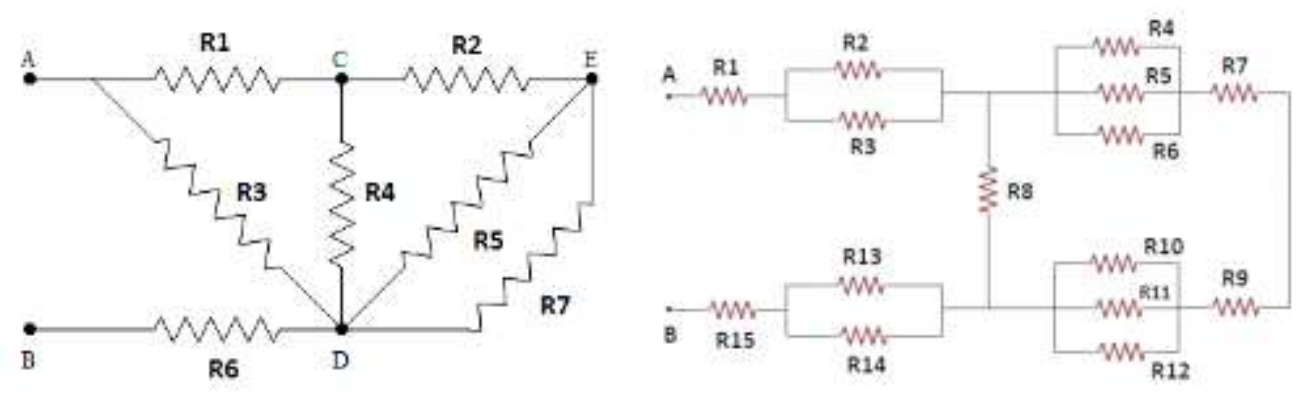

Fonte: Autores.

Através das montagens complexas, é possível explorar com melhor profundidade a aplicação das formulações matemáticas, bem como comprovar os resultados utilizando o ohmímetro proposto. Os alunos devem sugerir montagens e explorar aquelas desenvolvidas por outros grupos. Os dados de cada experimento devem ser coletados, depois tabulados em planilhas eletrônicas e armazenas para consultas subsequentes. Conforme sugere Haag et al., (2005) o tratamento de dados permite enriquecer as experiências de aprendizagem propiciando alternativas para o aluno compreender e relacionar os resultados obtidos e os conceitos vinculados à fundamentação teórica do experimento e, assim, trazer a Física escondida entre os números e fórmulas para o "mundo real".

Alunos e professores podem através do experimento, compartilhar soluções, comparar exemplos e sugerir novas abordagens, por exemplo:

a) alterar o resistor de calibração (Rr)

b) mesclar resistores de valores diferentes em um mesmo circuito

c) integrar montagens com outros grupos

d) comparar valores de circuitos iguais ou semelhantes

e) propor desafios para outros grupos de alunos

A diversidade de caminhos indicadas, permitem a exploração da plataforma Arduino agregada ao ensino de Física, como ferramenta de integração disciplinar tanto no âmbito escolar, quanto em pesquisas acadêmicas. Assim como instrumento introdutório a temas das ciências exatas e da natureza, lógica e aprendizagem ativa.

\section{Considerações Finais}

O presente trabalho teve como objetivo construir uma proposta de atividade experimental utilizando a placa de prototipagem Arduino, em aulas de eletricidade. Como visto anteriormente uma sequência didática foi sugerida a fim de criar passos para os alunos seguirem durante a aula, para que possa facilitar a programação do código de comando e familiarizar esses alunos, com o funcionamento do Arduino.

Na proposta de atividade experimental sugerida é possível trabalhar com os alunos a as Leis de Kirchhoff (Lei dos nós e Lei das malhas), expandido o aparato para outros conteúdos curriculares. O experimento ainda pode ser realizado em paralelo com um Objeto de Aprendizagem virtual, bem com um multímetro real. Alunos e professores amparados pelo instrumento podem aplicar, por fim, problemas encontrados/extraídos de livros didáticos do Ensino Fundamental ao Ensino Superior, perpassando o Ensino Médio, ou apostilas de ensino técnico, tonando a experiência do ensinar e do aprender factíveis e significativas, aproximando o saber sobre eletricidade à realidade vivenciada pelos aprendentes. Espera-se que o presente trabalho possa contribuir para que o uso do Arduino em aulas de física se torne cada vez mais crescente. 
Research, Society and Development, v. 10, n. 2, e11110212302, 2021

(CC BY 4.0) | ISSN 2525-3409 | DOI: http://dx.doi.org/10.33448/rsd-v10i2.12302

\section{Referências}

Assis, A. K. T. (2010). Os fundamentos experimentais e históricos da eletricidade. Montreal: Apeiron.

Banzi, M., \& Shiloh, M. (2011). Primeiros passos com o Arduino. São Paulo: Novatec, p1.

Brasil. Base Nacional Comum Curricular. Disponível em: http://basenacionalcomum. mec.gov.br/\#/site/base/o-que.

Brasil, S. (2000). Parâmetros curriculares nacionais: ensino médio. Ministério da Educação Brasília.

Brasil, \& MEC. (1999). Parâmetros curriculares nacionais para o ensino médio: PCNEM.

Brockington, G., \& Pietrocola, M. (2016). Serão as regras da Transposição Didática aplicáveis aos conceitos de Física Moderna? Investigações em Ensino de Ciências, 10(3), 387-404.

Chevallard, Y. (1991). La transposición didáctica. Del saber sabio al saber enseñado, 3.

da Rocha, F. S., \& Guadagnini, P. H. (2014). Projeto de um sensor de pressão manométrica para ensino de física em tempo real. Caderno Brasileiro de Ensino de Física, 31(1), 124-148.

da Rocha, F. S., Maranghello, G. F., \& Lucchese, M. M. (2014). Acelerômetro eletrônico e a placa Arduino para ensino de física em tempo real. Caderno Brasileiro de Ensino de Física, 31(1), 98-123.

Dorneles, P. F. T., Araujo, I. S., \& Veit, E. A. (2012). Integração entre atividades computacionais e experimentais como recurso instrucional no ensino de eletromagnetismo em física geral. Ciência \& Educação (Bauru), 18(1), 99-122.

Martins, L. Associação de Resistores. InfoEscola. <https://www.infoescola.com/fisica/associacao-de-resistores/>.

Maya, P. Á. (1979). Curso Básico de Eletricidade. (3a ed.), São Paulo: Distribuidora Cultura Brasileira LTDA.

Moreira, M. A. (2017). Grandes desafios para o ensino da física na educação contemporânea. Revista do Professor de Física, 1(1), 1-13.

Morosini, M. C. (2015). Estado de conhecimento e questões do campo científico. Educação (UFSM), 40(1), $101-116$.

Nardi, R. (2016). Memórias da educação em ciências no Brasil: a pesquisa em ensino de física. Investigações em Ensino de Ciências, $10(1)$, 63-101.

Robilotta, M. R. \& Babichak, C. C. (1997). Definições e conceitos em física. Cadernos Cedes, ano XVIII, 41, 35-45.

Silva, J. B. (2017). O contributo das tecnologias digitais para o ensino híbrido: o rompimento das fronteiras espaço-temporais historicamente estabelecidas e suas implicações no ensino. ARTEFACTUM-Revista de estudos em Linguagens e Tecnologia, 15(2).

Silva, J. B. da, Andrade, M. H., Oliveira, R. R. de, Sales, G. L., \& Alves, F. R. V. (2018). Tecnologias digitais e metodologias ativas na escola: o contributo do Kahoot para gamificar a sala de aula. Revista Thema, 15(2).

Sokoloff, D. R., Laws, P. W. \& Thornton, R. K. (2007). Real Time Physics: active learning labs transforming the introductory laboratory. European Journal of Physics, 28(3), 83-94.

Veiga, A. J. B. Proposta de currículo de nivelamento em física para cursos técnicos pós-médio noturnos da escola estadual Henrique Lage. 